although larger eyes such as Horse are tough at $4 \mu \mathrm{m}$ because of the size of the block. The larger eyes will be more successful if cut at 7-8 micrometers in thickness.

I expose into the area I want to section, then apply a gauze pad soaked in warm water and leave it for a few minutes. The lens will then be rehydrated enough to cooperate. The lens should look like glass in the ribbon.

When it all works well, eyes can be great fun. Tough to believe at this point, probably!

Mary Georger, Astra Arcus USA

\section{A Note On Electron Probe and SEM Filaments And Emission}

Electron Probe filaments are just the same as those used in the typical SEM. The difference is the position of the filament. If you place the filament a long way from the cap, you need less heat because the bias field is more effective. The result is less evaporation, less emission current and a longer filament life. You do not need many electrons to generate enough $\mathrm{x}$-rays for analysis compared with normal SEM imaging!

Saturation is saturation; it should be on the plateau of the graph. However the plateau may be moved higher or lower in the heat range depending on the position of the filament and the amount of bias being used. Don't write off electron gun importance. Probe current is basically the number of electrons: get more electrons from the gun and the current goes up. The gun sets the quality of an SEM image. Set the gun up incorrectly and the rest of the system cannot compensate, you just run out of electrons!

Driving the filament hard means pushing it forward and increasing the bias field to constrain the beam (aiming in a Japanese instrument for 100 to $120 \mu \mathrm{A}$ emission current). This filament pushed forward increases the number of electrons being emitted from the cap, but this means more heat is required to reach saturation as more heat is lost to the cathode cap, and because tha bias field effect is weakened. More heat shortens the filament life through evaporation. Increasing the bias constrains the electrons, funneling them together to try to achieve a small source. High performance requires a small, highly electron-dense source, which is improved further by using the correct anode-cathode distance of $1 \mathrm{~mm}$ for every $2 \mathrm{kV}$.

Put very simply, a $50 \mu \mathrm{m}$ source gives a $50 \AA$ microscope, the condenser system giving about a $10,000 \times$ reduction. Reduce the source size but keep up the number of electrons it constrains, and you improve the instrument's performance: a $40 \mu \mathrm{m}$ source gives a 40 À microscope. It is possible to get more than you paid for, but the cost is filament life.

Steve Chapman, Protrain

http://ourworld.compuserve.com/homepages/protrain

\section{Isopentane Freezing for $/ n$-Situ Hybridization}

For delicate tissues, especially those with a high water content (like soft embryonic tissue tends to be, at least, true for brain) that may be prone to cracking, I recommend freezing in isopentane cooled on dry ice.

Put the isopentane into a glass beaker and bury the beaker up to the liquid level in dry ice chunks.

Let it sit for 10 minutes or So, and I think the temperature is approximately $-50^{\circ} \mathrm{C}$. A little warmer than liquid $\mathrm{N}_{2}$ slush, but I think a bit difference in preventing cracking of that delicate tissue.

For our brains (adult rodent as well as embryonic), we have made a little foil "basket" with wire handles, and we dip the tissue in and out of the isopentane a few times before completely immersing it and leaving it for 20 to 30 seconds.

We freeze everything directly upon removal, without sucrose protection, and we generally have no problems with cracking. The cell morphology is, of course, not what I'm used to with perfusion-fixed material.

David Morilak, University of Texas Health Science Center

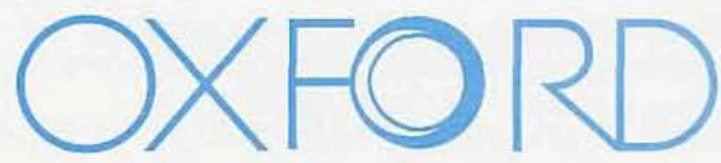

Oxford Instruments, Inc. Analytical Systems Division

\section{Applications Specialist}

Oxford Instruments Microanalysis Group, the leading supplier of $\mathrm{x}$-ray microanalysis equipment for electron microscopy, is seeking candidates for an entry level position as an Applications Specialist based in its US headquarters in Concord, Massachusetts.

This position provides technical support direct to customers and also support to our U.S. sales operation. Responsibilities would include customer training on Oxford equipment both onsite and also at our regularly scheduled, in house schools. Telephone support to customers with technical and software questions. Live and stand-alone demonstrations of products for sales purposes, including trade shows and roadshows.

Candidates must have experience in scanning electron microscopy and $x$-ray microanalysis, experience in transmission electron microscopy would be an advantage. A working knowledge on Windows based PC computers is essential. Candidates should ideally be educated to degree level in physical science or materials engineering with a strong chem. istry background.

Personal flexibility is required since this position includes widespread travel throughout the U.S. and possibly overseas.

\section{Field Support Engineer}

Oxford Instruments Customer Support Group, based in Concord, MA is looking for an individual living in the Northern Virginia - Metropolitan Washington, D.C. area to join them as a Field Support Engineer

In this position you will install, maintain, and support our customers in this area. Not only will you provide traditional field service, but you will also perform user training on both our products and applications, including Win 95 and Windows NT operating environments. Although most customers will be local, some overnight travel can be expected.

Both positions require good communication and administrative skills, combined with an outgoing and amiable personality. Candidates are expected to maintain a professional appearance.

\section{All applications must be received by 15 December 1997}

Interested candidates should mail their resumes to:

Oxford Instruments America, Inc.

Atten.: Human Resources

130A Baker Avenue Ext.

Concord, MA 01742

Or may FAX their resumes to: (508)287-5097

Or may eMail their resumes to: kindlan@oxford.usa.com

Oxford Instruments America, Inc. is an EOE/AA Employer 